2011 Shanghai Asia-Pacific School and Workshop on Gravitation International Journal of Modern Physics: Conference Series

Vol. 7 (2012) 194-201

(C) World Scientific Publishing Company

DOI: $10.1142 / \mathrm{S} 2010194512004266$

\title{
DARK ENERGY AND QCD GHOST
}

\author{
NOBUYOSHI OHTA \\ Department of Physics, Kinki University \\ Higashi-Osaka, Osaka 577-8502, Japan \\ ohtan@phys.kindai.ac.jp
}

\begin{abstract}
We argue that the dark energy that explains the observed accelerating expansion of the universe may arise due to the contribution to the vacuum energy of the QCD ghost in a time-dependent background. We show that the QCD ghost produces dark energy proportional to the Hubble parameter $H \Lambda_{Q C D}^{3}\left(\Lambda_{Q C D}\right.$ is the QCD mass scale) which has the right magnitude $\sim\left(3 \times 10^{-3} \mathrm{eV}\right)^{4}$.
\end{abstract}

Keywords: Dark Energy; QCD ghost; Hubble parameter.

PACS numbers: 11.25.Hf, 123.1K

\section{Introduction}

The recent cosmological observations have confirmed the existence of the early inflationary epoch and the accelerated expansion of the present universe. ${ }^{1,2}$ Observational result is consistent with the picture that the universe has the dark energy, about $75 \%$ of the total energy density. The simplest possibility is the vacuum energy or cosmological constant whose origin is yet to be identified.

Such vacuum energy is easily incorporated in the quantum field theory. The vacuum fluctuations in quantum field theory naturally induce such a vacuum energy, but the problem is how to control the size of it. The contribution of quantum fluctuations in known fields up to $300 \mathrm{GeV}$, which is about the highest energy at which the current theories have been verified, gives a vacuum energy density of order $(300 \mathrm{GeV})^{4}$. This is vastly larger than the observed dark energy density $\left(3 \times 10^{-3}\right.$ $\mathrm{eV})^{4}$ by a factor of order $10^{56}$. Assuming the tree-level contribution is zero, it is a great challenge how to understand the origin of this tiny energy density.

Recently a very interesting suggestion on the origin of a cosmological term is made, without introducing new degrees of freedom beyond what are already known, with the cosmological constant of just the right magnitude to give the observed expansion. ${ }^{4}$ We claim that the cosmological term arises from the contribution of the ghost fields which are supposed to be present in the low-energy effective theory of QCD. ${ }^{5,6,7,8,9}$ The ghosts are required to exist for the resolution of the $\mathrm{U}(1)$ problem, but are completely decoupled from the physical sector. ${ }^{9}$ The above claim is that the ghosts are decoupled from the physical states and make no contribution 
in the flat Minkowski space, but once they are in the curved space or time-dependent background, the cancellation of their contribution to the vacuum energy is off-set, leaving a small energy density $\rho \sim H \Lambda_{Q C D}^{3}$, where $H$ is the Hubble parameter and $\Lambda_{Q C D}$ is the QCD mass scale of order a hundred MeV. With $H \sim 10^{-33} \mathrm{eV}$, this gives the right magnitude.

Originally it was formulated in a toy model similar to the Schwinger model (proposed by Kogut and Susskind ${ }^{10}$ ) using two scalar fields of positive and negative norms. These scalar fields cancel with each other, leaving no trace in the physical subspace in the flat Minkowski space, but it is argued that they have small contribution to the vacuum energy in the curved space or time-dependent background. (Similar system is used in a mechanism of supersymmetry breaking in Ref. 11.) However it is known that the QCD ghost must be intrinsically vector field in order for the U(1) problem to be consistently resolved within the framework of QCD. ${ }^{12}$ We show that this mechanism works even if we keep all the modes of the vector ghost. We then find that the result is factor 2 larger.

It is interesting to note that the proposed 'cosmological constant' is proportional to the Hubble parameter $H(t)$ but not its square, and thus it is T-violating. This is because the origin of this term has that property. It is extremely interesting to see what physical effects this may have on the evolution of our universe.

In the next section, we briefly recapitulate how the $\mathrm{U}(1)$ problem is resolved by the vector ghost following Ref. 9, and also show how the ghost decouples from the physical sector. In sect. 3, following the discussions in Ref. 13, we discuss the mechanism of generating a tiny contribution to the vacuum energy in the Rindler space as a typical example of the time-dependent background. ${ }^{14,15}$ We argue that due to the change of the definition of the vacuum, we indeed obtain small contribution to the vacuum energy proportional to the Hubble parameter. We find that our result is factor 2 larger than the previous estimate. Sect. 4 is devoted to the discussions and conclusions.

\section{Decoupling of the vector ghost in the Minkowski space}

Let us consider the low-energy effective Lagrangian ${ }^{7,8,9}$

$$
\mathcal{L}=\frac{1}{2}\left(\partial_{\mu} S\right)^{2}-\frac{1}{2} m_{N S}^{2} S^{2}+\frac{1}{2 F_{S}^{2}\left(m_{S}^{2}-m_{N S}^{2}\right)}\left(\partial_{\mu} K^{\mu}\right)^{2}-\frac{1}{F_{S}} S \partial_{\mu} K^{\mu}
$$

where $S$ is a flavor-singlet pseudoscalar field with the decay constant $F_{S}, K^{\mu}$ is an axial vector "field", which in QCD corresponds to the gluonic current

$$
K^{\mu}=2 N_{f} \frac{g^{2}}{16 \pi^{2}} \epsilon^{\mu \nu \lambda \sigma} A_{\nu}^{a}\left(\partial_{\lambda} A_{\sigma}^{a}+\frac{1}{3} g f^{a b c} A_{\lambda}^{b} A_{\sigma}^{c}\right),
$$

where $N_{f}$ is the number of flavors.

The Lagrangian (1) is a relevant part of more realistic model for the low-energy effective theory for meson system reflecting the QCD properties. It is invariant 
under the gauge transformation

$$
K^{\mu} \rightarrow K^{\mu}+\epsilon^{\mu \nu \lambda \sigma} \partial_{\nu} \Lambda_{\lambda \sigma},
$$

where $\Lambda_{\lambda \sigma}$ denotes an arbitrary antisymmetric tensor. This transformation reflects the color gauge invariance of the underlying QCD. ${ }^{9,12}$

To quantize this system, we break the gauge invariance under (3) by adding the term

$$
\frac{1}{4 F_{S}^{2}\left(m_{S}^{2}-m_{N S}^{2}\right) \alpha}\left(\partial_{\mu} K_{\nu}-\partial_{\nu} K_{\mu}\right)^{2},
$$

where $\alpha$ is a gauge parameter. The simplest case is to choose $\alpha=1$. One can then derive the Feynman rules as follows:

$$
\begin{gathered}
K_{\mu} \text {-propagator: } \frac{i \eta_{\mu \nu}}{k^{2}} F_{S}^{2}\left(m_{S}^{2}-m_{N S}^{2}\right), \quad S \text {-propagator: } \frac{i}{k^{2}-m_{N S}^{2}}, \\
S-K_{\mu} \text {-mixing: } \frac{1}{F_{S}} k_{\mu} .
\end{gathered}
$$

It appears that the system (1) describes a scalar field $S$ with mass $m_{N S}$ and a massless vector. However it is not difficult to show that the mass of the scalar $S$ gets shifted to $m_{S}$ due to the mixing of the scalar and vector modes. This is the approach in Ref. 6. Alternatively, in the general gauge, one can derive the two-point functions

$$
\begin{aligned}
T\left\langle K_{\mu}(x) K_{\nu}(y)\right\rangle_{M}= & \int \frac{d^{4} k}{(2 \pi)^{4}} e^{-i k \cdot(x-y)} i\left\{\frac{k^{2}-m_{N S}^{2}}{k^{2}-m_{S}^{2}} \frac{k_{\mu} k_{\nu}}{\left(k^{2}\right)^{2}}\right. \\
& \left.+\frac{\alpha}{k^{2}}\left(\eta_{\mu \nu}-\frac{k_{\mu} k_{\nu}}{k^{2}}\right)\right\} F_{S}^{2}\left(m_{S}^{2}-m_{N S}^{2}\right), \\
T\langle S(x) S(y)\rangle_{M}= & \int \frac{d^{4} k}{(2 \pi)^{4}} e^{-i k \cdot(x-y)} \frac{i}{k^{2}-m_{S}^{2}}, \\
T\left\langle S(x) K_{\mu}(y)\right\rangle_{M}= & \int \frac{d^{4} k}{(2 \pi)^{4}} e^{-i k \cdot(x-y)} \frac{F_{S}\left(m_{S}^{2}-m_{N S}^{2}\right)}{k^{2}\left(k^{2}-m_{S}^{2}\right)},
\end{aligned}
$$

where $T$ denotes the time-order and the subscript $M$ stand for the expectation value by the Minkowski vacuum. We see that $S$ has the shifted mass $m_{S}$ instead of the original $m_{N S}$, confirming the above claim. This is one of the consequences of the massless mode $K_{\mu}$ and gives a resolution of the $U(1)$ problem. What happens to the massless mode in the system? Because we observe no massless field in the low-energy world, it must decouple from the physical sector. We now show how this can be achieved in the Minkowski space.

The field equations from the Lagrangian (1) are

$$
\begin{aligned}
& \square K_{\mu}-\partial_{\mu}\left\{(1-\alpha) \partial^{\nu} K_{\nu}+F_{S}\left(m_{S}^{2}-m_{N S}^{2}\right) S\right\}=0, \\
& \square S+m_{N S}^{2} S+\frac{1}{F_{S}} \partial^{\mu} K_{\mu}=0 .
\end{aligned}
$$

From the first equation in (7), we find

$$
\square\left(\partial_{\mu} K_{\nu}-\partial_{\nu} K_{\mu}\right)=0 .
$$


Hence we can consistently impose the subsidiary condition on the physical states:

$$
\left.\left(\partial_{\mu} K_{\nu}-\partial_{\nu} K_{\mu}\right)^{(+)} \mid \text {phys }\right\rangle=0 .
$$

In the gauge $\alpha=1$, we can write the mode expansion of the vector field. In terms of the canonically normalized field $K_{\mu}^{\prime}(x) \equiv F_{S} \sqrt{m_{S}^{2}-m_{N S}^{2}} K_{\mu}(x)$, it takes the form

$$
K_{\mu}^{\prime}(x)=\int \frac{d^{3} \boldsymbol{k}}{(2 \pi)^{3 / 2} \sqrt{2 k_{0}}} e_{\mu}^{(\lambda)}\left[e^{-i k \cdot(x-y)} a(\boldsymbol{k}, \lambda)+e^{i k \cdot(x-y)} a^{\dagger}(\boldsymbol{k}, \lambda)\right],
$$

where $e_{\mu}^{(1)}=\left(0, e^{(1)}\right)$ and $e_{\mu}^{(2)}=\left(0, e^{(2)}\right)$ represent the transverse modes, $e_{\mu}^{(3)}=$ $\left(0, \boldsymbol{e}^{(3)}\right)$ the longitudinal mode, and $e_{\mu}^{(0)}=(1,0)$ the time component with

$$
e^{(3)}=\frac{k}{|k|}, \quad e^{(1)} \cdot e^{(2)}=e^{(1)} \cdot e^{(3)}=e^{(2)} \cdot e^{(3)}=0
$$

Canonical quantization of the system then gives

$$
\left[a(\boldsymbol{k}, \lambda), a^{\dagger}\left(\boldsymbol{k}^{\prime}, \lambda^{\prime}\right)\right]=\eta_{\lambda \lambda^{\prime}} \delta^{3}\left(\boldsymbol{k}-\boldsymbol{k}^{\prime}\right),
$$

We find that the transverse modes have the opposite sign to the usual gauge fields. The condition (9) means that the two transverse components $a(\boldsymbol{k}, 1), a(\boldsymbol{k}, 2)$ and the combination $\frac{1}{\sqrt{2}}[a(\boldsymbol{k}, 3)-a(\boldsymbol{k}, 0)]$ should annihilate the physical state. This forbids the states generated by the two transverse components and by $\frac{1}{\sqrt{2}}\left[a^{\dagger}(\boldsymbol{k}, 3)+\right.$ $\left.a^{\dagger}(\boldsymbol{k}, 0)\right]$. The remaining component $\frac{1}{\sqrt{2}}\left[a^{\dagger}(\boldsymbol{k}, 3)-a^{\dagger}(\boldsymbol{k}, 0)\right]$ can only produce zero norm states, so that all the components of $K_{\mu}$ are completely decoupled from the physical state. Nevertheless it produces the physical effect of shifting the mass of the singlet pseudoscalar $S$ and resolves the problem associated with the $\eta^{\prime}$ meson decay. ${ }^{9}$ In the Kogut-Susskind model, similar subsidiary condition can be imposed. ${ }^{4}$ This mechanism was also discussed in the context of spontaneous breakdown of supersymmetry. ${ }^{11}$

\section{Vector ghost in the Rindler space}

We now turn to the QCD vector ghost in the Rindler space. Consider the Minkowski space

$$
d s^{2}=d t^{2}-d x^{2}-d y^{2}-d z^{2} \equiv d \bar{u} d \bar{v}-d x^{2}-d y^{2},
$$

where we have defined

$$
\bar{u}=t-z, \quad \bar{v}=t+z .
$$

Under the transformation

$$
t=\frac{1}{a} e^{a \xi} \sinh a \eta, \quad z=\frac{1}{a} e^{a \xi} \cos a \eta, \quad(-\infty<\eta, \xi<\infty, a>0),
$$

we obtain

$$
\begin{gathered}
d s^{2}=e^{a(v-u)} d u d v-d x^{2}-d y^{2}=e^{2 a \xi}\left(d \eta^{2}-d \xi^{2}\right)-d x^{2}-d y^{2}, \\
\bar{u}=-\frac{1}{a} e^{a(\xi-\eta)} \equiv-\frac{1}{a} e^{-a u}, \quad \bar{v}=\frac{1}{a} e^{a(\xi+\eta)} \equiv \frac{1}{a} e^{a v},
\end{gathered}
$$


The Rindler coordinates $\eta$ and $\xi$ in (15) describe only the quadrant part $z>|t|$ called R. The opposite quadrant part L: $z<-|t|$ is described by changing the signs in (15). The rest of our Minkowski space are described by analytic continuation of these coordinates. ${ }^{14,15}$

The wave function to be used in our massless vector field can be obtained from the solutions for the scalar wave equation $\phi_{; \alpha}^{; \alpha}=0$. Explicitly this becomes in our coordinate system $\left[e^{-2 a \xi}\left(\partial_{\eta}^{2}-\partial_{\xi}^{2}\right)-\partial_{x}^{2}-\partial_{y}^{2}\right] \phi=0$. We denote by ${ }^{R} u(\boldsymbol{k})$ the wave function which asymptotes

$$
R u(\boldsymbol{k})=\left\{\begin{array}{lll}
e^{-i k_{0} u} e^{-i\left(k_{1} x+k_{2} y\right)} & \text { in } \mathrm{R} \\
0 & \text { in } \mathrm{L}
\end{array}\right.
$$

along the surface $v=-\infty, \bar{v}=0$, the past horizon of the Rindler coordinate. Similarly the wave function in the $\mathrm{L}$ region is defined as

$$
{ }^{L} u(\boldsymbol{k})=\left\{\begin{array}{lll}
0 & \text { in } \mathrm{R} \\
e^{i k_{0} v} e^{-i\left(k_{1} x+k_{2} y\right)} & \text { in } \mathrm{L}
\end{array}\right.
$$

The positive-frequency Minkowski modes are characterized by the condition that they are analytic and bounded in the lower half complex $\bar{u}$ plane on $\bar{v}=0$. The combinations

$$
\begin{aligned}
& \frac{1}{\left[2 \sinh \left(\pi k_{0} /(2 a)\right)\right]^{1 / 2}}\left(e^{\pi k_{0} /(2 a) R} u(\boldsymbol{k})+e^{-\pi k_{0} /(2 a) L} u(-\boldsymbol{k})^{*}\right), \\
& \frac{1}{\left[2 \sinh \left(\pi k_{0} /(2 a)\right)\right]^{1 / 2}}\left(e^{-\pi k_{0} /(2 a) R} u(-\boldsymbol{k})^{*}+e^{\pi k_{0} /(2 a) L} u(\boldsymbol{k})\right),
\end{aligned}
$$

where ${ }^{L} u(-\boldsymbol{k})$ and ${ }^{R} u(-\boldsymbol{k})$ denote the wave function with minus momenta, have precisely this property, ${ }^{14,15}$ so we can use these modes to express our Minkowski space field:

$$
\begin{aligned}
K_{\mu}^{\prime}(x)= & \int \frac{d^{3} \boldsymbol{k}}{(2 \pi)^{3 / 2} \sqrt{2 k_{0}}} \frac{e_{\mu}^{(\lambda)}}{\left.2 \sinh \left(\pi k_{0} /(2 a)\right)\right]^{1 / 2}} \\
& {\left[\left(e^{\pi k_{0} /(2 a) R} u(\boldsymbol{k})+e^{-\pi k_{0} /(2 a) L} u(-\boldsymbol{k})^{*}\right) a^{(1)}(\boldsymbol{k}, \lambda)\right.} \\
& \left.+\left(e^{-\pi k_{0} /(2 a) R} u(-\boldsymbol{k})^{*}+e^{\pi k_{0} /(2 a) L} u(\boldsymbol{k})\right) a^{(2)}(\boldsymbol{k}, \lambda)+\text { h.c. }\right],
\end{aligned}
$$

and the Minkowski vacuum is defined as

$$
a^{(i)}(\boldsymbol{k}, 1)\left|0_{M}\right\rangle=a^{(i)}(\boldsymbol{k}, 2)\left|0_{M}\right\rangle=\left[a^{(i)}(\boldsymbol{k}, 3)-a^{(i)}(\boldsymbol{k}, 0)\right]\left|0_{M}\right\rangle=0, \quad(i=1,2) .(2
$$

The field in the Rindler space is written as

$$
\begin{aligned}
K_{\mu}^{\prime}(x)=\int & \frac{d^{3} \boldsymbol{k}}{(2 \pi)^{3 / 2} \sqrt{2 k_{0}}} e_{\mu}^{(\lambda)}\left[{ }^{L} u(\boldsymbol{k}) b^{(1)}(\boldsymbol{k}, \lambda)+{ }^{L} u(\boldsymbol{k})^{*} b^{(1) \dagger}(\boldsymbol{k}, \lambda)\right. \\
& \left.+{ }^{R} u(\boldsymbol{k}) b^{(2)}(\boldsymbol{k}, \lambda)+{ }^{R} u(\boldsymbol{k})^{*} b^{(2) \dagger}(\boldsymbol{k}, \lambda)\right]
\end{aligned}
$$


Comparing (21) and (23), we see that

$$
\begin{aligned}
& b^{(1)}(\boldsymbol{k}, \lambda)=\frac{1}{\sqrt{2 \sinh \frac{\pi k_{0}}{a}}}\left[e^{\pi k_{0} /(2 a)} a^{(2)}(\boldsymbol{k}, \lambda)+e^{-\pi k_{0} /(2 a)} a^{(1) \dagger}(-\boldsymbol{k}, \lambda)\right], \\
& b^{(2)}(\boldsymbol{k}, \lambda)=\frac{1}{\sqrt{2 \sinh \frac{\pi k_{0}}{a}}}\left[e^{\pi k_{0} /(2 a)} a^{(1)}(\boldsymbol{k}, \lambda)+e^{-\pi k_{0} /(2 a)} a^{(2) \dagger}(-\boldsymbol{k}, \lambda)\right] .
\end{aligned}
$$

The resulting energy for each mode is then given by

$$
\begin{aligned}
& \left\langle 0_{M}\left|\int d^{3} \boldsymbol{k}^{\prime} \sum_{\lambda, \lambda^{\prime}=0}^{3} k_{0} \eta_{\lambda \lambda^{\prime}} b^{(1) \dagger}(\boldsymbol{k}, \lambda) b^{(1)}\left(\boldsymbol{k}^{\prime}, \lambda^{\prime}\right)\right| 0_{M}\right\rangle \\
& =\left\langle 0_{M}\left|\int d^{3} \boldsymbol{k}^{\prime} \sum_{\lambda, \lambda^{\prime}=0}^{3} k_{0} \eta_{\lambda \lambda^{\prime}} b^{(2) \dagger}(\boldsymbol{k}, \lambda) b^{(2)}\left(\boldsymbol{k}^{\prime}, \lambda^{\prime}\right)\right| 0_{M}\right\rangle=\frac{4 k_{0}}{e^{2 \pi k_{0} / a}-1} .
\end{aligned}
$$

The vector field has four degrees of freedom and all of them are decoupled in the flat Minkowski space. However, we find that they all contribute to the vacuum energy in the Rindler space. This is somewhat surprising because naively one would expect that the longitudinal and scalar modes would cancel. Here instead of cancelling, they add up. This is the main result. The result is factor 2 different from that in Ref. 4, but the order of magnitude is the same.

One may ask why such a difference occurred and whether the disagreement is resolved if we consider the Faddeev-Popov (FP) ghost. It is argued that the ghost does not make any contribution. ${ }^{13}$ The difference comes not from this but from the treatment of the transverse modes. It was assumed there that the transverse modes decouple and make no contribution, so they were completely eliminated from the outset. This is true for the Minkowski space, but the whole point of the discussions is that the result and cancellation are modified when the system is considered in the time-dependent background. So eliminating these modes from the start cannot be justified. Thus it is expected that all four components contribute to the dark energy.

The contribution of high frequency modes is suppressed by the factor $e^{-2 \pi k_{0} / a}$ and the main contribution comes from $k_{0} \sim a$. In the cosmological context, $a \sim H$ and hence $k_{0} \sim H$, giving the vacuum energy proportional to the Hubble parameter. In the context of strongly interacting confining QCD with topological nontrivial sector, this effect occurs only in the time direction and their wave function in other space directions is expected to have the size of QCD energy scale. As a result, this ghost gives the vacuum energy density $H \Lambda_{Q C D}^{3}$ of the right magnitude $\sim\left(3 \times 10^{-3}\right.$ $\mathrm{eV})^{4}$. Thus this vacuum energy arises due to the mismatch between the vacuum energies computed in slowly expanding universe and Minkowski space. The effect is infrared in nature. The local physics with $k_{0} \gg a$ is not affected by the unphysical modes with high accuracy. 


\section{Discussions and conclusions}

Here, correcting the argument of Ref. 4 in accordance with QCD, we have first clarified the decoupling mechanism of the QCD vector ghost in the flat Minkowski space, and then evaluated the contribution of the QCD vector ghost to the vacuum energy density in the Rindler space as a typical example of time-dependent spacetime, and found that it gives the vacuum energy proportional to the Hubble parameter.

This model has extremely interesting feature. First of all, this does not assume new degrees of freedom only to produce nonzero cosmological constant. Rather it is induced by the already existing field just because the universe is expanding. Secondly it gives the amazing result of the cosmological constant of right magnitude without artificial fine tuning. Note that the vacuum energy is not just a constant but depends on the Hubble parameter.

One may wonder that the unphysical modes or polarization of QED photon may also contribute to the dark energy of the similar amount. However, QED is weakly interacting theory unlike QCD. So we expect that we do not need to consider them. ${ }^{4}$

Other possible origin of such a "vacuum energy" was also suggested based on the QCD condensate, ${ }^{16}$ assuming that there is no contribution for the flat Minkowski spacetime. Our mechanism is different in that such an assumption is not necessary.

It has been suggested that the same ghost may also generate magnetic field in an expanding universe. ${ }^{17}$ This is also discussed keeping only two components of the QCD vector ghost. It would be interesting to check if this mechanism makes sense with the vector ghost. Another interesting question is to try to find if there is any other effects to check the proposed mechanism. There are already some discussions on this type of dark energy. ${ }^{18,19,20,21,22,23,24,25}$

We analyzed the physical states and the resulting contribution to the cosmological term in the Rindler space as a typical example of time-dependent space. However, it does not give so realistic model. It is thus interesting to study the mechanism in more realistic model of Friedmann-Lemaître-Robertson-Walker universe.

\section{Acknowledgement}

I am grateful for valuable discussions with R.-G. Cai, H. Kodama, K. Maeda and F. R. Urban, and the organizers of 2011 Shanghai Asia-Pacific School and Workshop on Gravitation for their warm hospitality where this work is presented. This work was supported in part by the Grant-in-Aid for Scientific Research Fund of the JSPS (C) No. 20540283, No. 21.09225 and (A) No. 22244030.

\section{References}

1. A. G. Riess et al. [Supernova Search Team Collaboration], Astron. J. 116 (1998) 1009 [arXiv:astro-ph/9805201].

2. D. N. Spergel et al. [WMAP Collaboration], Determination of Cosmological Parameters," Astrophys. J. Suppl. 148 (2003) 175 [arXiv:astro-ph/0302209].

3. N. Ohta, Phys. Lett. B 695 (2011) 41 [arXiv:1010.1339 [astro-ph.CO]]. 
4. F. R. Urban and A. R. Zhitnitsky, Phys. Lett. B 688 (2010) 9 [arXiv:0906.2162 [grqc]]; Phys. Rev. D 80 (2009) 063001 [arXiv:0906.2165 [hep-th]]; JCAP 0909 (2009) 018 [arXiv:0906.3546 [astro-ph.CO]]; Nucl. Phys. B 835 (2010) 135 [arXiv:0909.2684 [astro-ph.CO]].

5. E. Witten, Nucl. Phys. B 156 (1979) 269.

6. G. Veneziano, Nucl. Phys. B 159 (1979) 213.

7. C. Rosenzweig, J. Schechter and C. G. Trahern, Phys. Rev. D 21 (1980) 3388.

8. P. Nath and R. L. Arnowitt, Phys. Rev. D 23 (1981) 473.

9. K. Kawarabayashi and N. Ohta, Nucl. Phys. B 175 (1980) 477; Prog. Theor. Phys. 66 (1981) 1789; N. Ohta, Prog. Theor. Phys. 66 (1981) 1408.

10. J. B. Kogut and L. Susskind, Phys. Rev. D 11 (1975) 3594.

11. N. Ohta, Phys. Lett. B 112 (1982) 215; N. Ohta and Y. Fujii, Nucl. Phys. B 202 (1982) 477; N. Ohta, H. Sugata and H. Yamaguchi, Phys. Rev. D 30 (1984) 2181; N. Ohta, Phys. Rev. D 32 (1985) 1467.

12. H. Hata, T. Kugo and N. Ohta, Nucl. Phys. B 178 (1981) 527.

13. A. R. Zhitnitsky, Phys. Rev. D 82 (2010) 103520 [arXiv:1004.2040 [gr-qc]].

14. W. G. Unruh, Phys. Rev. D 14 (1976) 870.

15. N. D. Birrell and P. C. W. Davies, "Quantum Fields In Curved Space," Cambridge Univ. Press (1982) 340p.

16. R. Schutzhold, Phys. Rev. Lett. 89 (2002) 081302; F. R. Klinkhamer and G. E. Volovik, Phys. Rev. D 79 (2009) 063527 [arXiv:0811.4347 [gr-qc]].

17. F. R. Urban and A. R. Zhitnitsky, Phys. Rev. D 82 (2010) 043524 [arXiv:0912.3248 [astro-ph.CO]].

18. S. Basilakos, M. Plionis and J. Sola, Phys. Rev. D 80 (2009) 083511 [arXiv:0907.4555 [astro-ph.CO]].

19. W. Zimdahl, H. A. Borges, S. Carneiro, J. C. Fabris and W. S. Hipolito-Ricaldi, JCAP 1104 (2011) 028 [arXiv:1009.0672 [astro-ph.CO]].

20. R. G. Cai, Q. Su, Z. L. Tuo and H. B. Zhang, arXiv:1011.3212 [astro-ph.CO].

21. B. Holdom, Phys. Lett. B 697 (2011) 351 [arXiv:1012.0551 [hep-ph]].

22. J. B. Jimenez and A. L. Maroto, magnetic fields," arXiv:1101.2072 [astro-ph.CO].

23. A. Sheykhi and M. S. Movahed, arXiv:1104.4713 [hep-th].

24. A. Sheykhi and A. Bagheri, arXiv:1104.5271 [physics.gen-ph].

25. C. J. Feng, X. Z. Li and X. Y. Shen, arXiv:1105.3253 [hep-th]. 